\title{
Mathematical Model for Electric Field Sensor Based on Whispering Gallery Modes Using Navier's Equation for Linear Elasticity
}

\author{
Amir R. Ali ${ }^{1,2}$ and Mohamed A. Kamel ${ }^{1}$ \\ ${ }^{1}$ Applied-Science \& Robotics Laboratory for Applied-Mechatronics (ARAtronics Lab.), Mechatronics Engineering Department, \\ German University in Cairo, New Cairo, Cairo 11835, Egypt \\ ${ }^{2}$ Micro-Sensor Laboratory, Mechanical Engineering Department, Southern Methodist University, Dallas, TX 75275, USA
}

Correspondence should be addressed to Amir R. Ali; arahmed@mail.smu.edu

Received 30 March 2017; Revised 5 June 2017; Accepted 18 June 2017; Published 25 July 2017

Academic Editor: Constantin Fetecau

Copyright (C) 2017 Amir R. Ali and Mohamed A. Kamel. This is an open access article distributed under the Creative Commons Attribution License, which permits unrestricted use, distribution, and reproduction in any medium, provided the original work is properly cited.

\begin{abstract}
This paper presents and verifies the mathematical model of an electric field senor based on the whispering gallery mode (WGM). The sensing element is a dielectric microsphere, where the light is used to tune the optical modes of the microsphere. The light undergoes total internal reflection along the circumference of the sphere; then it experiences optical resonance. The WGM are monitored as sharp dips on the transmission spectrum. These modes are very sensitive to morphology changes of the sphere, such that, for every minute change in the sphere's morphology, a shift in the transmission spectrum will happen and that is known as WGM shifts. Due to the electrostriction effect, the applied electric field will induce forces acting on the surface of the dielectric sphere. In turn, these forces will deform the sphere causing shifts in its WGM spectrum. The applied electric field can be obtained by calculating these shifts. Navier's equation for linear elasticity is used to model the deformation of the sphere to find the WGM shift. The finite element numerical studies are performed to verify the introduced model and to study the behavior of the sensor at different values of microspheres' Young's modulus and dielectric constant. Furthermore, the sensitivity and resolution of the developed WGM electric filed sensor model will be presented in this paper.
\end{abstract}

\section{Introduction}

Recently, optical cavities have been used for many sensory applications due their high quality factor and resolution $[1,2]$. These applications include wall shear stress calculation $[3,4]$; temperature measurement [5-7]; biosensing [8-13]; hemozoin nanocrystals detection in aquatic environment [14]; nanoparticle detection [15]; and acceleration measurement [16-18]. In this paper, WGM based optical cavities are used to detect and measure the applied electric field. These cavities come in different shapes such as sphere, toroid, disk, and cylinder. The optical modes of these cavities can be excited using tunable laser. A single mode optical fiber is utilized to couple the incoming laser light into the cavity. When resonance occurs, the WGM modes are monitored as sharp dips on the transmission. WGM modes are highly responsive to changes in the morphology of the cavity and the outer medium; consequently, they are also called morphology dependent resonances (MDR) modes. Any minute external disturbance on the sphere or its surrounding environment will be detected as shift in its transmission spectrum. Consequently, these cavities are used in many sensory applications by measuring the WGM shifts corresponding to the change in the measured phenomenon.

Figure 1 presents a microsphere WGM cavity of an outer radius $b$ subjected to an applied electric field of strength $E_{0}$. The sphere is usually fabricated from dielectric polymeric material, like polydimethylsiloxane (PDMS). At the beginning, an optical fiber ( $5 \mathrm{~cm}$ long) was stripped of its plastic cladding and then subjected to heat while stretching. The final diameter size after stretching is $\sim 50 \mu \mathrm{m}$. Afterwards, the fiber is dipped into the PDMS polymer pool. Then, a sphere 


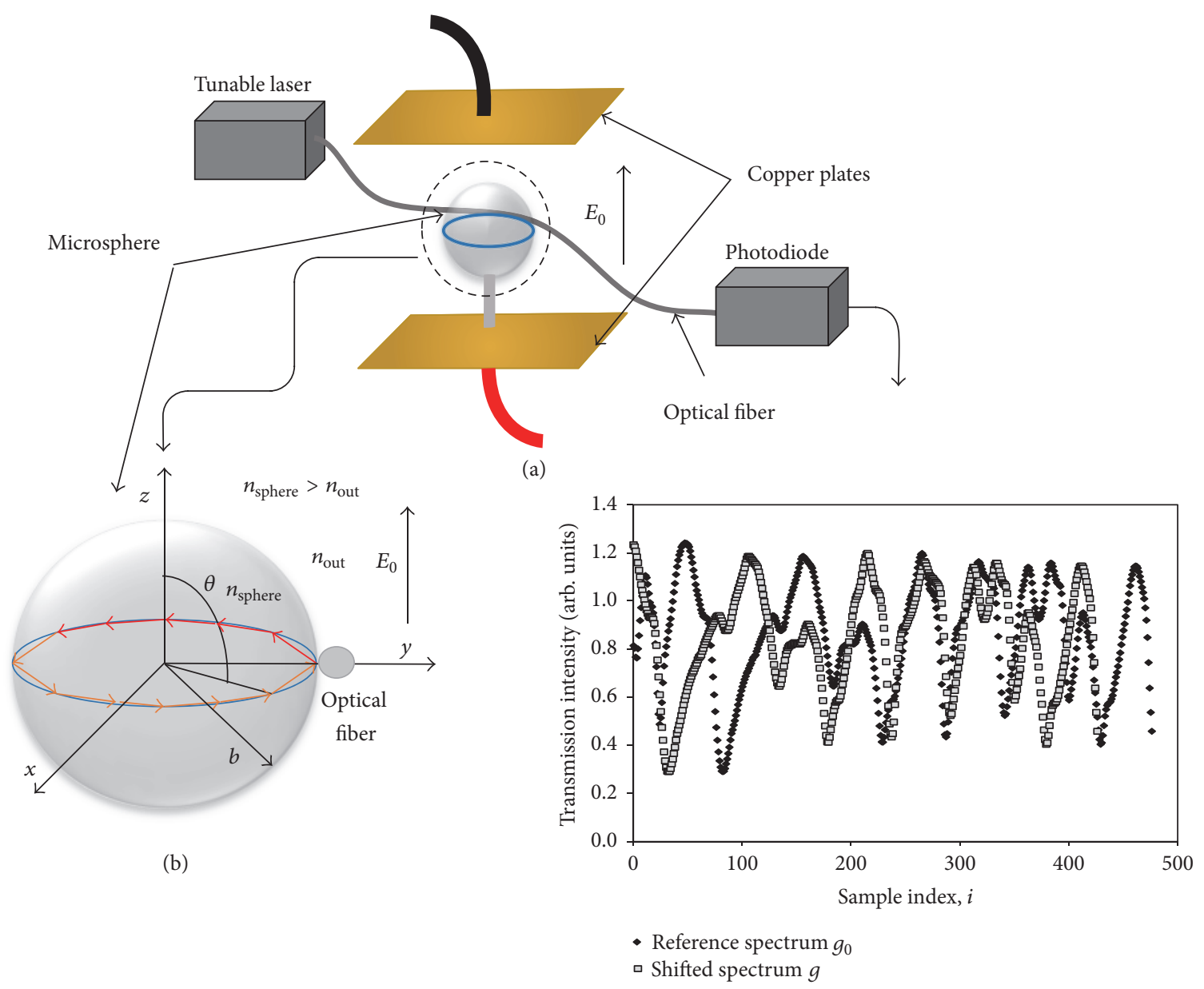

(c)

FIGURE 1: (a) Schematic diagram of the WGM based electric field sensor, (b) WGM microsphere, and (c) transmission spectrum for a microsphere before and after morphology changes.

of PDMS will form at the end of the fiber due to the surface tension and the gravitational forces. The fiber and sphere assembly is heated to cure of the polymeric material as reported in [19].

The microsphere is coupled to a single mode optical fiber. A tunable laser is utilized to supply the light traveling through the optical fiber. The light is coupled to a microsphere cavity through the tapered section of the optical fiber; then it circumnavigates the microsphere undergoing total internal reflection. The condition of total internal reflection must be fulfilled, such that the refractive index of the cavity $n_{\text {sphere }}$ is greater than the surrounding $n_{\text {out }}$. A photodiode is placed on the other side of the optical fiber to monitor the intensity of the transmitted light.

The optical resonance will only happen when the total distance traveled by the light (round trip) is almost equal to a multiple integer $k$ of the light's wavelength as seen in 1st-order approximation of (1). Then the WGM would be presented as a sharp dips on the transmission spectrum as shown in Figure 1(c).

$$
2 \pi n_{\text {sphere }} b \approx k \lambda, \quad b \gg \lambda
$$

where $n_{\text {sphere }}$ is the refractive index the microsphere; $\lambda$ is the wavelength of the light; and $b$ is the radius of the sphere. The change in the morphology (size or the refractive index) of the cavity will induce a shift $d \lambda$ in the resonance wavelength as follows:

$$
\frac{d \lambda}{\lambda}=\frac{d b}{b}+\frac{d n_{\text {sphere }}}{n_{\text {sphere }}} .
$$

In this paper, the microsphere is used to measure the applied electric field due to the electrostriction effect. When an electric field is applied on a dielectric microsphere, it induces surface and body forces across its body. In turn, theses forces will deform the sphere changing it size, causing shifts in its WGM transmission spectrum, thus creating a WGM based electric field sensor. The induced electrostrictive volumetric body force $\vec{f}$ can be written as follows [20]:

$$
\vec{f}=-\frac{1}{2} E^{2} \nabla \varepsilon_{\text {sphere }}-\frac{1}{4}\left(a_{1}+a_{2}\right) \nabla E^{2},
$$

where $\varepsilon_{\text {sphere }}$ is the dielectric constant of the microsphere's material; $E$ is the applied electric field inside the sphere; and 
$a_{1}$ and $a_{2}$ are coefficients describing the strain of the dielectric material due to the applied electric field in the directions parallel and perpendicular to the field. $\vec{f}$ is the summation of the gradients of $E^{2}$ and $\varepsilon_{\text {sphere }}$. For a homogenous dielectric material under a static uniform applied electric field, both gradients of (3) are equal to zero; thus $\vec{f}$ is also zero. However, at the interface separating the microsphere and the surrounding, a surface pressure force $\vec{P}$ exists. It exists due to the discontinuity of the dielectric constant along the media and given by

$$
\begin{aligned}
\vec{P}= & {[\alpha \vec{E}(\vec{E} \cdot \vec{n})]_{o}+[\alpha \vec{E}(\vec{E} \cdot \vec{n})]_{s}-\left[\beta E^{2} \vec{n}\right]_{o} } \\
& -\left[\beta E^{2} \vec{n}\right]_{s} .
\end{aligned}
$$

$\alpha$ and $\beta$ are coefficients that depend on the electrostrictive properties of the microsphere's material. $\vec{n}$ is the unit surface normal vector and the subscripts indicate the values to be taken on either side of the interface ( $s$ denotes the sphere's and $o$ denotes the outer medium). The induced deformation due to the pressure forces can be obtained by solving Navier's equation for linear elasticity at steady state, which is given by

$$
\nabla^{2} \vec{u}+\frac{1}{1-2 \nu} \nabla(\nabla \cdot \vec{u})+\frac{\vec{f}}{G}=0,
$$

where $\vec{u}$ is the deformation of the cavity, $v$ is Poisson's ratio, and $G$ is the shear modulus of the cavity's material. Thus the deformation of the microsphere needs to be calculated to get the WGM shift due to the applied electric field.

\section{Analysis}

2.1. Electric Field and Pressure Force Calculation. To solve Navier's equation, the pressure forces must be obtained first; so the first step is to calculate the electric field composing the pressure forces. The applied electric field is obtained by differentiating the electric potential. When a dielectric microsphere is placed under an applied static axisymmetric electric field, the potential is defined by Laplace's equation in spherical coordinates $(r, \theta, \varphi)[21]$.

$$
\nabla^{2} D=0
$$

where $D$ is the electric potential. Due to the symmetry of the applied electric field, the solution of Laplace's equation is also independent of the azimuthal angle $\varphi$. Separation of variables method is used to obtain the solution $D(r, \theta)$.

$$
D(r, \theta)=\sum_{m=0} r^{m}\left(A_{m}+\frac{B_{m}}{r}\right) P_{m}(\cos \theta)
$$

where $r$ is radius of the microsphere, $\theta$ is the azimuthal angle between the position vector and the $z$ axis, $P_{m}$ is the associated Legendre polynomial of the $m$ th order, and $A_{m}$, $B_{m}$ are coefficients. The boundary conditions enforced on the microsphere, due the continuity of the potential and electric field lines at the interface (where the value of the radius $r$ is equal to $b$ ), are

$$
\begin{gathered}
D_{\text {sphere }}(b)=D_{\text {out }}(b), \\
\varepsilon_{\text {sphere }} \frac{\partial D_{\text {sphere }}}{\partial r}=\left.\varepsilon_{\text {out }} \frac{\partial D_{\text {out }}}{\partial r}\right|_{r=b} .
\end{gathered}
$$

$D_{\text {sphere }}$ and $D_{\text {out }}$ are the electric potential inside and outside the microsphere, respectively. $\varepsilon_{\text {out }}$ is the dielectric constant of material outside the sphere. After the application of the boundary conditions, the coefficients are $A_{m}$ and $B_{m}$ are obtained. The gradient of the potential is applied to find the radial $E_{r}$ and azimuthal $E_{\theta}$ components of the applied electric field inside and outside the cavity. The electric field components are

$$
E_{r}= \begin{cases}\frac{-3 E_{0} \varepsilon_{\text {out }}}{2 \varepsilon_{\text {out }}+\varepsilon_{\text {sphere }}} \cos \theta & r<b \\ -E_{0}\left(\frac{2 b^{3}\left(\varepsilon_{\text {out }}-\varepsilon_{\text {sphere }}\right)}{r^{3}\left(2 \varepsilon_{\text {out }}+\varepsilon_{\text {sphere }}\right)}-1\right) \cos \theta & r>b,\end{cases}
$$

$E_{\theta}$

$$
= \begin{cases}\frac{3 E_{0} \varepsilon_{\text {out }}}{\varepsilon_{\text {sphere }}+2 \varepsilon_{\text {out }}} \sin \theta & r<b \\ E_{0}\left(\frac{b^{3}\left(\varepsilon_{\text {out }}-\varepsilon_{\text {sphere }}\right)+r^{3}\left(2 \varepsilon_{\text {out }}+\varepsilon_{\text {sphere }}\right)}{r^{3}\left(2 \varepsilon_{\text {out }}+\varepsilon_{\text {sphere }}\right)}\right) \cos \theta & r>b .\end{cases}
$$

$E_{0}$ is the applied electric field strength. The acting radial and azimuthal components of the pressure force $\left(P_{r}\right.$ and $\left.P_{\theta}\right)$ on the interface are

$$
\begin{aligned}
P_{r} & =\frac{9 E_{0}^{2}}{4\left(2 \varepsilon_{\text {out }}+\varepsilon_{\text {sphere }}\right)^{2}}\left(( a _ { 1 } + a _ { 2 } + 2 \varepsilon _ { 0 } - \varepsilon _ { \text { out } } ) \left(\varepsilon_{\text {sphere }}\right.\right. \\
& \left.-\varepsilon_{\text {out }}\right)^{2}+\left(\left(a_{1}+a_{2}+2 \varepsilon_{0}\right) \varepsilon_{\text {sphere }}\right. \\
& \left.+\left(a_{1}+a_{2}+2 \varepsilon_{0}-\varepsilon_{\text {out }}\right) \varepsilon_{\text {out }}+\varepsilon_{\text {out }}^{2}\right)\left(\varepsilon_{\text {sphere }}-\varepsilon_{\text {out }}\right) \\
& \cdot \cos 2 \theta), \\
P_{\theta}= & \frac{-9 E_{0}^{2}\left(a_{1}+2 \varepsilon_{0}\right)\left(\varepsilon_{\text {sphere }}-\varepsilon_{\text {out }}\right) \varepsilon_{\text {out }} \sin 2 \theta}{4\left(2 \varepsilon_{\text {out }}+\varepsilon_{\text {sphere }}\right)^{2}} .
\end{aligned}
$$

$\varepsilon_{0}$ is the dielectric permittivity of vacuum $(8.854 \times$ $\left.10^{-12} \mathrm{~F} \cdot \mathrm{m}^{-1}\right)$. The boundary stresses acting on the sphere will be equated to the pressure forces. These forces will deform the microsphere; and the deformation is calculated using Navier's equation.

2.2. Solution of Navier's Equation. Due to the absence of the body force, Navier's equation can be written as follows:

$$
\nabla^{2} \vec{u}+\frac{1}{1-2 \nu} \nabla(\nabla \cdot \vec{u})=0 .
$$

Using Helmholtz theory, $\vec{u}$ can be expressed as follows [24]:

$$
\vec{u}=\vec{u}_{s}+\vec{u}_{\mathrm{ir}}
$$


where $\vec{u}_{s}$ and $\vec{u}_{\text {ir }}$ are solenoidal and irrotational vectors, respectively; these vectors satisfy the following relations:

$$
\begin{gathered}
\nabla \times \vec{u}_{\text {ir }}=0, \\
\nabla \cdot \vec{u}_{s}=0 .
\end{gathered}
$$

Thus $\vec{u}$ can be written as follows:

$$
\vec{u}=\vec{B}+\nabla \Gamma
$$

where $\vec{B}$ is a harmonic vector; and $\Gamma$ is a scalar potential function. Substitution of (14) in (11) yields

$$
\nabla^{2} \Gamma=\frac{-1}{2(1-v)} \nabla \cdot \nabla \vec{B}
$$

$\Gamma$ is chosen to satisfy the previous equation and given by

$$
\Gamma=\frac{-1}{4(1-v)}\left(\vec{R} \cdot \vec{B}+B_{0}\right)
$$

where $B_{0}$ is harmonic and satisfying the boundary conditions; and $\vec{R}$ is the position vector. After substitution of $\Gamma$ in (11), the solution to Navier's equation in spherical coordinates can be written as follows:

$$
\vec{u}=4(1-v) \vec{B}-\nabla\left(\vec{R} \cdot \vec{B}+B_{0}\right) .
$$

Laplace's equation is solved again to find the harmonic vector $\vec{B}$, where the components of $\vec{B}$ and $B_{0}$ are represented as a series of Legendre polynomials. Thus, the radial deformation of the sphere due to the applied electric field can be written as follows:

$$
\begin{array}{r}
u_{r} \\
=\sum_{n}\left[A_{n}(n+1)(n-2+4 v) R^{n+1}+B_{n} n R^{n-1}\right] P_{n}(\xi) \\
\xi=\cos (\theta) .
\end{array}
$$

$u_{r}$ is series of the associated Legendre polynomials $P_{n}(\xi)$ of the $n$th order. The polynomials are multiplied by the coefficients $A_{n}$ and $B_{n}$. These coefficients are also obtained using the boundary conditions imposed on the sphere. Finally the boundary stresses in the radial $\sigma_{r r}$ and azimuthal $\sigma_{r \theta}$ directions derived from the deformations are represented as follows:

$$
\begin{aligned}
\sigma_{r r} & =2 G \sum_{n}\left[A_{n}(n+1)\left(n^{2}-n-2-2 v\right) R^{n}\right. \\
& \left.+B_{n} n(n-1) R^{n-2}\right] P_{n}(\xi), \\
\sigma_{r \theta} & =2 G \sum_{n}\left[A_{n}\left(n^{2}+2 n-1+2 v\right) R^{n}\right. \\
& \left.+B_{n}(n-1) R^{n-2}\right] \frac{d}{d \theta} P_{n}(\xi) .
\end{aligned}
$$

In electrostriction, the pressure forces are the applied boundary stresses on the microsphere and satisfy

$$
\begin{gathered}
\sigma_{r r}=\left.P_{r}\right|_{r=b} \\
\sigma_{r \theta}=\left.P_{\theta}\right|_{r=b} .
\end{gathered}
$$

In this case the solution $\vec{u}$ can be presented as follows:

$$
\begin{aligned}
u_{r} & =\frac{3 b E_{0}{ }^{2}\left(\varepsilon_{\text {sphere }}-\varepsilon_{\text {out }}\right)}{4 E_{e}\left(\varepsilon_{\text {sphere }}+2 \varepsilon_{\text {out }}\right)^{2}(1+v)^{2}}\left(\left(\left(a_{1}+a_{2}+2 \varepsilon_{0}\right)\right.\right. \\
& \left.\cdot \varepsilon_{\text {sphere }}+\left(a_{1}+a_{2}+2 \varepsilon_{0}-\varepsilon_{\text {sphere }}\right) \varepsilon_{\text {out }}-2 \varepsilon_{\text {out }}^{2}\right)(4 \\
& -2 v)+\frac{(1+v)}{7+5 v}\left(-7\left(a_{1}+a_{2}+2 \varepsilon_{0}\right) \varepsilon_{\text {sphere }}-7\left(a_{1}\right.\right. \\
& \left.+a_{2}+2 \varepsilon_{0}-\varepsilon_{\text {sphere }}\right) \varepsilon_{\text {out }}-7 \varepsilon_{\text {out }}^{2} \\
& +2 v\left(2\left(a_{1}+a_{2}+2 \varepsilon_{0}\right) \varepsilon_{\text {sphere }}\right. \\
& \left.-\left(25 a_{1}-2 a_{2}+50 \varepsilon_{0}+2 \varepsilon_{\text {sphere }}\right) \varepsilon_{\text {out }}+2 \varepsilon_{\text {out }}^{2}\right)(1 \\
& +3 \cos (2 \theta)))) .
\end{aligned}
$$

$E_{e}$ is Young's modulus of the material of the microsphere. The radial deformation $u_{r}$ is directly proportional to the square of the electric field strength; inversely proportional to $E_{e}$; and dependent on the dielectric constants $\varepsilon_{\text {sphere }}$ and $\varepsilon_{\text {out }}$. Navier's equation is solved at the equator $(\theta$ is $\pi / 2)$; thus, the calculated $u_{r}$ is the same change $d r$ in the optical path of the circumnavigating light:

$$
\begin{aligned}
u_{r} & =\frac{9 b E_{0}{ }^{2}\left(\varepsilon_{\text {sphere }}-\varepsilon_{\text {out }}\right)}{2 E_{e}\left(\varepsilon_{\text {sphere }}+2 \varepsilon_{\text {out }}\right)^{2}(1+v)^{2}(7+5 v)}\left(2 v \left(a_{1}\right.\right. \\
& \left.+a_{2}+2 \varepsilon_{0}\right)(2+v) \varepsilon_{\text {sphere }}+2 v \varepsilon_{\text {out }}\left(11 a_{1}+2 a_{2}\right. \\
& \left.+22 \varepsilon_{0}-2 \varepsilon_{\text {sphere }}+v\left(10 a_{1}+a_{2}+20 \varepsilon_{0}-\varepsilon_{\text {sphere }}\right)\right) \\
& \left.+\varepsilon_{\text {out }}^{2}(7-v(5+8 v))\right) .
\end{aligned}
$$

The $d \lambda$ is independent of the outer radius $b$ and can be written using (2) as follows:

$$
\begin{aligned}
d \lambda & =\frac{9 \lambda_{L} E_{0}{ }^{2}\left(\varepsilon_{\text {sphere }}-\varepsilon_{\text {out }}\right)}{2 E_{e}\left(\varepsilon_{\text {sphere }}+2 \varepsilon_{\text {out }}\right)^{2}(1+v)^{2}(7+5 v)}\left(2 v \left(a_{1}\right.\right. \\
& \left.+a_{2}+2 \varepsilon_{0}\right)(2+v) \varepsilon_{\text {sphere }}+2 v \varepsilon_{\text {out }}\left(11 a_{1}+2 a_{2}\right. \\
& \left.+22 \varepsilon_{0}-2 \varepsilon_{\text {sphere }}+v\left(10 a_{1}+a_{2}+20 \varepsilon_{0}-\varepsilon_{\text {sphere }}\right)\right) \\
& \left.+\varepsilon_{\text {out }}^{2}(7-v(5+8 v))\right) .
\end{aligned}
$$

$\lambda_{L}$ is the wavelength of the light provided by the tunable laser. Using Navier's equation, an optical WGM based electric field sensor can be constructed. The relation between the microsphere's WGM shift and the applied electric field is investigated extensively by means of numerical studies using finite element simulation software. 
TABLE 1: Simulation parameters for the 1st numerical study.

\begin{tabular}{lc}
\hline Parameter & Magnitude \\
\hline Microsphere (PDMS 60:1) & $600 \mu \mathrm{m}$ \\
Diameter & 0.49 \\
Poisson's ratio & $1030 \mathrm{~kg} / \mathrm{m}^{3}$ \\
Density & $2.7 \times \varepsilon_{0}$ \\
Dielectric constant & $3 \mathrm{kpa}$ \\
Young's modulus & \\
\hline Outer medium (air) & $1 \times \varepsilon_{0}$ \\
$\quad$ Dielectric constant & \\
\hline Laser light (infrared) & $1.312 \mu \mathrm{m}$ \\
\hline
\end{tabular}

2.3. Numerical Studies. Three numerical studies are done to investigate the relation between $d \lambda$ and the applied electric field. The studies calculate $d \lambda$ using the deformation of the sphere due to the electrostriction effect. The purpose of the first two studies is to confirm the quadratic dependence of sphere's WGM shift on the applied electric field strength and the inverse proportionality between the sphere's WGM shift and Young's modulus of the sphere's material, while the third one aims to see the effect varying the dielectric constant of the sphere on its WGM shift. During the studies, the electric field is supplied by means of 2 copper electrodes. The microsphere is situated at the center between the plates as seen in Figure 1.

For the first study, cured PDMS [25] is used as the construction material of the microsphere. The mixture has an elastomer to curing agent ratio of $60: 1$. Under steadystate conditions, $d \lambda$ is measured against an increasing applied electric field. The simulation parameters during the first study are presented in Table 1.

As for the second study, the inverse proportionality between the sphere's WGM shift and Young's modulus of the sphere's material is investigated. The study measures $d \lambda$ of several microspheres with different Young's moduli under the same applied electric field strength. The simulation parameters of the second study is similar to the first one, except for the varying Young's modulus value. Finally, the third study investigates the effect of varying the sphere's dielectric constant on its WGM shift. The 3rd study subjects several microspheres with different values of dielectric constant to the same applied electric field input; then it measures the value of $d \lambda$ of the several microspheres. Also, the simulation parameters of the 3rd study is similar to the first one, except for the changing dielectric constant. The strength of the applied electric field for the $2 \mathrm{nd}$ and $3 \mathrm{rd}$ studies is $5 \mathrm{kV} / \mathrm{m}$.

\section{Results and Discussion}

Results of the 1st numerical studies are shown in Figure 2. $d \lambda$ is plotted against the increasing electric field strength up to $1300 \mathrm{kV} / \mathrm{m}$. The increasing electric field will be met by the increasing shift. It can be seen that the quadratic relation between $d \lambda$ and the electric field strength is confirmed. In Figure 2, the numerical range under study was split to three main regions $(0-200 \mathrm{kV} / \mathrm{m}, 200-500 \mathrm{kV} / \mathrm{m}$, and $500-1300 \mathrm{kV} / \mathrm{m})$. It is clear that the nonlinear behavior in the response was linearized per each region to be able to find the linear relation between the output $d \lambda$ and the input $E_{0}$. Since the proposed sensor is a linear optical sensor, so we will find out the sensitivity and the resolution for that sensor per each region. Table 2 summarized sensitivity and resolution values for each mentioned region.

Figure 3 shows the results of the second study, where the WGM shift $d \lambda$ is modeled against the varying Young's modulus $\left(E_{e}\right)$ of microsphere for different values of applied electric field strength $(0.5 \mathrm{kV} / \mathrm{m}, 1 \mathrm{kV} / \mathrm{m}, 1.5 \mathrm{kV} / \mathrm{m}, 2 \mathrm{kV} / \mathrm{m}$, and $5 \mathrm{kV} / \mathrm{m}$ ). Decreasing Young's modulus of the sphere will lead to the increase of the shift. It is clear that the WGM shift is inversely proportional with respect to Young's modulus of the sensing element material.

Finally, Figure 4 shows the results of the 3rd study, which is the WGM shift of the microsphere is directly proportional to the relative dielectric constant $\left(\varepsilon_{R}\right)$ of the microsphere. In this study, we do find the sensor response for the same applied electric field strength mentioned in the 2 nd study $(0.5 \mathrm{kV} / \mathrm{m}$, $1 \mathrm{kV} / \mathrm{m}, 1.5 \mathrm{kV} / \mathrm{m}, 2 \mathrm{kV} / \mathrm{m}$, and $5 \mathrm{kV} / \mathrm{m}$ ).

Up to now, several sensor technologies for electric field detection have been implemented. Table 3 summarizes the sensitivities of electric field from various techniques. In this paper, we show that our new modeling technique will be promising to be used in a complex sensor structure like multilayered composite sphere as reported in [26].

\section{Conclusion}

In this paper, the mathematical model of a WGM based electric field senor is presented. Navier's equation for linear elasticity is solved to find the deformation of the seeing element upon application of the electric field strength with different values $(0.5 \mathrm{kV} / \mathrm{m}, 1 \mathrm{kV} / \mathrm{m}, 1.5 \mathrm{kV} / \mathrm{m}, 2 \mathrm{kV} / \mathrm{m}$, and $5 \mathrm{kV} / \mathrm{m}$ ). The response indicates that the WGM shift in the transmission spectrum has a quadratic dependence on the applied electric field strength. However, the linearization behavior for the sensor is needed for the practical usage. That was the reason to split the sensing range into three main regions $(0-200 \mathrm{kV} / \mathrm{m}, 200-500 \mathrm{kV} / \mathrm{m}$, and $500-1300 \mathrm{kV} / \mathrm{m})$. Results show that the WGM shift is inversely proportional to Young's modulus of the microsphere's material under different electric field strength values $(0.5 \mathrm{kV} / \mathrm{m}, 1 \mathrm{kV} / \mathrm{m}, 1.5 \mathrm{kV} / \mathrm{m}$, $2 \mathrm{kV} / \mathrm{m}$, and $5 \mathrm{kV} / \mathrm{m}$ ). Furthermore, the studies also show that the WGM shift is directly proportional to the dielectric constant of the microsphere's material under the same electric filed strength values that were mentioned before. The sensor behavior and characteristics were verified by numerical finite element studies. Finally, in this paper, we show that our new promising modeling technique will be easily used in a complex sensor structure like multilayered composite sphere. 
TABLE 2: Sensitivity and resolution along the different detection regions.

\begin{tabular}{lccc}
\hline & $0-200 \mathrm{kV} / \mathrm{m}$ & $200-500 \mathrm{kV} / \mathrm{m}$ & $500-1300 \mathrm{kV} / \mathrm{m}$ \\
\hline Sensitivity $\left|d \lambda / d E_{0}\right| \mathrm{nm} /(\mathrm{kV} / \mathrm{m})$ & 0.00014 & 0.00048 & 0.00119 \\
Resolution $\mathrm{kV} / \mathrm{m}$ & 28.64 & 23.17 & 57.88 \\
\hline
\end{tabular}

TABLE 3: Electric field sensor's sensitivity with different techniques.

\begin{tabular}{lcrr}
\hline Electric field detection technique & Sensitivity achieved & Reference & Year \\
\hline Unpolarized PDMS sphere & $8 \times 10^{-4} \mathrm{pm} /(\mathrm{V} / \mathrm{m})$ & {$[22]$} & 2012 \\
Polarized PDMS sphere & $6 \times 10^{-3} \mathrm{pm} /(\mathrm{V} / \mathrm{m})$ & {$[22]$} & 2012 \\
Beam coupled PDMS cavity & $0.01 \mathrm{pm} /(\mathrm{V} / \mathrm{m})$ & {$[22]$} & 2012 \\
PDMS homogenous sphere & $0.0017 \mathrm{pm} /(\mathrm{V} / \mathrm{m})$ & {$[23]$} & 2014 \\
Composite sphere & $0.027 \mathrm{pm} /(\mathrm{V} / \mathrm{m})$ & {$[23]$} & 2014 \\
\hline
\end{tabular}

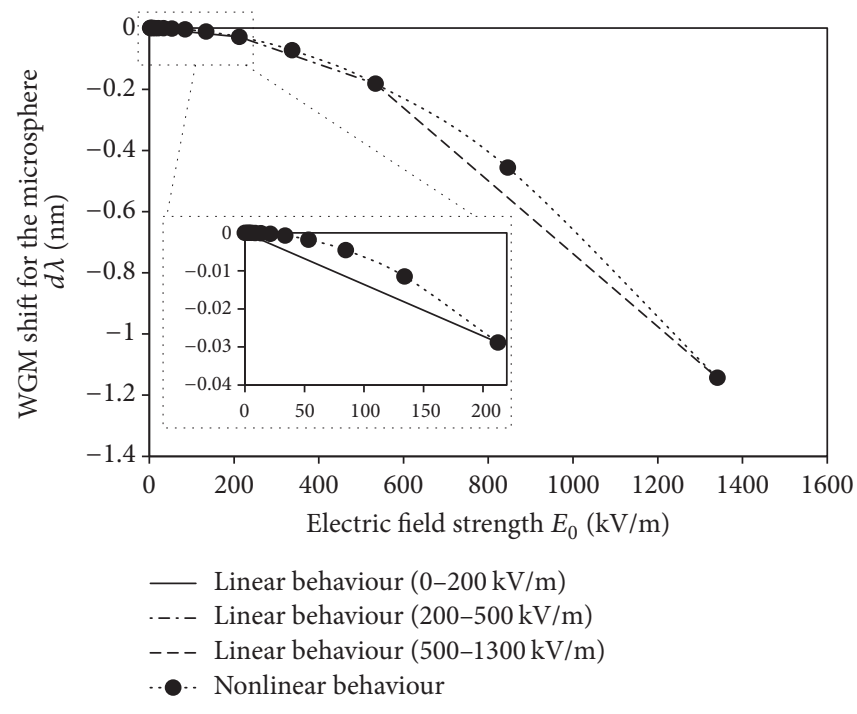

FIGURE 2: WGM shifts $d \lambda$ against the applied electric field strength during the 1st study.

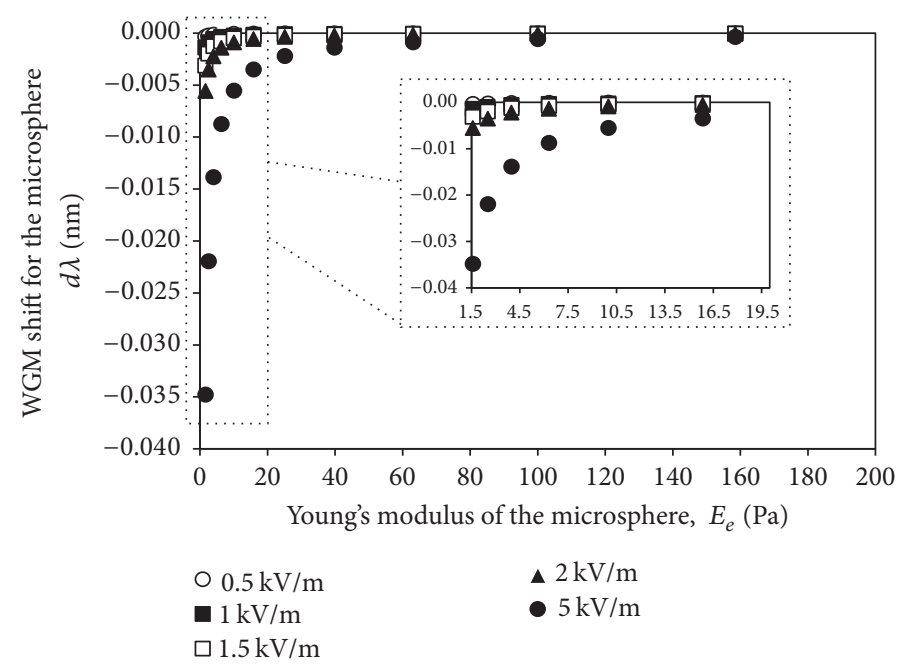

Figure 3: WGM shifts $d \lambda$ against Young's modulus during the 2nd study. 


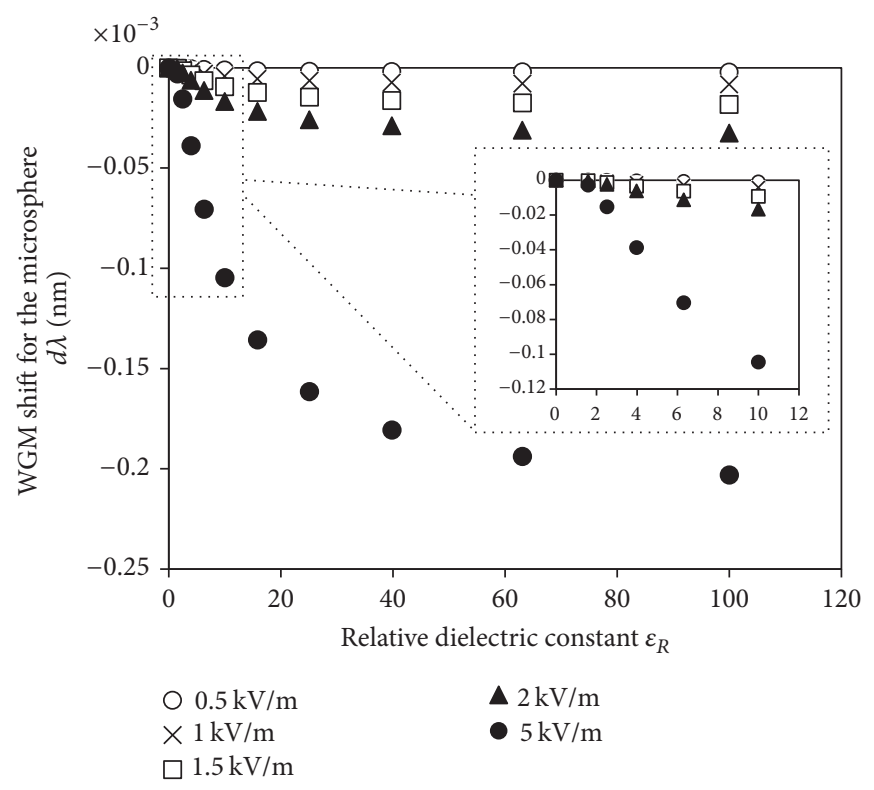

FIgURE 4: WGM shifts $d \lambda$ against relative dielectric constant during the 3 rd study.

\section{Conflicts of Interest}

The authors declare that there are no conflicts of interest regarding the publication of this paper.

\section{References}

[1] M. L. Gorodetsky, A. A. Savchenkov, and V. S. Ilchenko, "Ultimate Q of optical microsphere resonators," Optics Letters, vol. 21, no. 7, pp. 453-455, 1996.

[2] F. Monifi, Ş. Kaya Özdemir, and L. Yang, "Tunable add-drop filter using an active whispering gallery mode microcavity," Applied Physics Letters, vol. 103, no. 18, Article ID 181103, 2013.

[3] U. K. Ayaz, T. Ioppolo, and M. V. Ötügen, "Direct measurement of wall shear stress in a reattaching flow with a photonic sensor," Measurement Science and Technology, vol. 24, no. 12, Article ID 124001, 2013.

[4] U. K. Ayaz, T. Ioppolo, and M. V. Ötügen, "Wall shear stress sensor based on the optical resonances of dielectric microspheres," Measurement Science and Technology, vol. 22, no. 7, Article ID 075203, 2011.

[5] B. Özel, R. Nett, T. Weigel, G. Schweiger, and A. Ostendorf, "Temperature sensing by using whispering gallery modes with hollow core fibers," Measurement Science and Technology, vol. 21, no. 9, Article ID 094015, 2010.

[6] G. Guan, S. Arnold, and M. V. Otugen, "Temperature measurements using a microoptical sensor based on whispering gallery modes," AIAA Journal, vol. 44, no. 10, pp. 2385-2389, 2006.

[7] C. H. Dong, L. He, Y. F. Xiao et al., "Fabrication of high- Q polydimethylsiloxane optical microspheres for thermal sensing," Applied Physics Letters, vol. 94, no. 23, pp. 1-3, 2009.

[8] L. He, Ş. K. Özdemir, J. Zhu, W. Kim, and L. Yang, "Detecting single viruses and nanoparticles using whispering gallery microlasers," Nature Nanotechnology, vol. 6, no. 7, pp. 428-432, 2011.

[9] F. Vollmer, S. Arnold, D. Braun, I. Teraoka, and A. Libchaber, "Multiplexed DNA quantification by spectroscopic shift of two microsphere cavities," Biophysical Journal, vol. 85, no. 3, pp. 1974-1979, 2003.

[10] K. Schult, A. Katerkamp, D. Trau, F. Grawe, K. Cammann, and M. Meusel, "Disposable optical sensor chip for medical diagnostics: New ways in bioanalysis," Analytical Chemistry, vol. 71, no. 23, pp. 5430-5435, 1999.

[11] S. Arnold and S. I. Shopova, "Whispering Gallery Mode Biosensor," in Biophotonics: Spectroscopy, Imaging, Sensing, and Manipulation, NATO Science for Peace and Security Series B: Physics and Biophysics, pp. 237-259, Springer, Dordrecht, The Netherlands, 2011.

[12] F. Vollmer and S. Arnold, "Whispering-gallery-mode biosensing: label-free detection down to single molecules," Nature Methods, vol. 5, no. 7, pp. 591-596, 2008.

[13] W. Lukosz, "Integrated optical chemical and direct biochemical sensors," Sensors and Actuators B: Chemical, vol. 29, no. 1-3, pp. 37-50, 1995.

[14] W. Kim, S. K. Ozdemir, J. Zhu, M. Faraz, C. Coban, and L. Yang, "Detection and size measurement of individual hemozoin nanocrystals in aquatic environment using a whispering gallery mode resonator," Optics Express, vol. 20, no. 28, pp. 2942629446, 2012.

[15] Ş. K. Özdemir, J. Zhu, X. Yang et al., "Highly sensitive detection of nanoparticles with a self-referenced and self-heterodyned whispering-gallery Raman microlaser," Proceedings of the National Academy of Sciences of the United States of America, vol. 111, no. 37, pp. E3836-E3844, 2014.

[16] A. B. Matsko, A. A. Savchenkov, V. S. Ilchenko, and L. Maleki, "Optical gyroscope with whispering gallery mode optical cavities," Optics Communications, vol. 233, no. 1-3, pp. 107-112, 2004.

[17] T. Ioppolo, V. Ötügen, D. Fourguette, and L. Larocque, "Effect of acceleration on the morphology-dependent optical resonances of spherical resonators," Journal of the Optical Society of America B: Optical Physics, vol. 28, no. 2, pp. 225-227, 2011.

[18] J.-P. Laine, C. Tapalian, B. Little, and H. Haus, "Acceleration sensor based on high-Q optical microsphere resonator and 
pedestal antiresonant reflecting waveguide coupler," Sensors and Actuators A: Physical, vol. 93, no. 1, pp. 1-7, 2001.

[19] T. Ioppolo, V. Ötügen, and U. Ayaz, "Development of whispering gallery mode polymeric micro-optical electric field sensors," Journal of Visualized Experiments, vol. 71, no. e50199, 2013.

[20] J. A. Stratton, Electromagnetic Theory, Mcgraw-Hill Book Company, New York and London, 1941.

[21] P. Lorrain, D. R. Corson, and F. Lorrain, Electromagnetic Fields and Waves Including Electric Circuits, Freeman and Company, 1988.

[22] A. R. Ali, T. Ioppolo, and M. V. Otugen, "High-resolution electric field sensor based on whispering gallery modes of a beamcoupled dielectric resonator," in Proceedings of the 1st International Conference on Engineering and Technology, ICET 2012, Cairo, Egypt, October 2012.

[23] A. R. Ali, T. Ioppolo, V. Ötügen, M. Christensen, and D. MacFarlane, "Photonic electric field sensor based on polymeric microspheres," Journal of Polymer Science, Part B: Polymer Physics, vol. 52, no. 3, pp. 276-279, 2014.

[24] R. W. Soutas-Little, Elasticity, Dover Publications, 1999.

[25] Dow Corning, "Sylgard ${ }^{\circledR} 184$ Silicone Elastomer," 1-3184B-01 data sheet, April 2014.

[26] A. R. Ali and M. A. Kamel, "Novel techniques for optical sensor using single core multilayer structures for electric field detection," in Proceedings of SPIE 10231, Prague, Czech Republic, May 2017. 


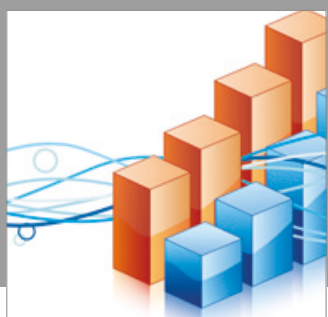

Advances in

Operations Research

vatersals

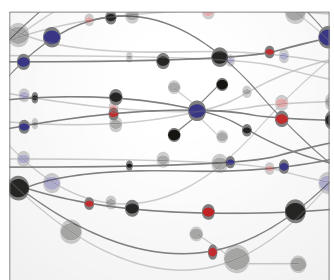

\section{The Scientific} World Journal
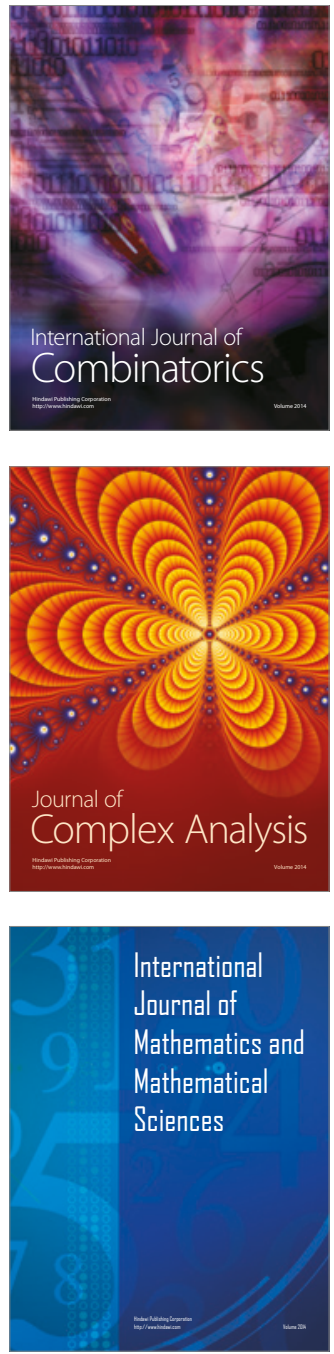
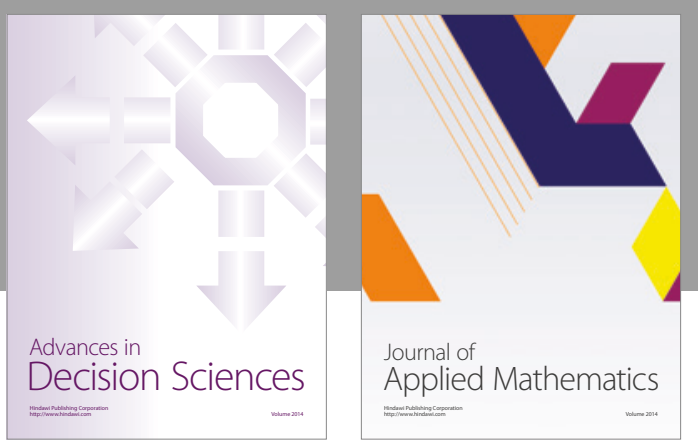

Algebra

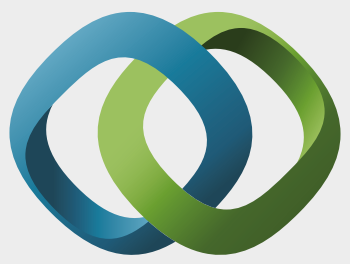

\section{Hindawi}

Submit your manuscripts at

https://www.hindawi.com
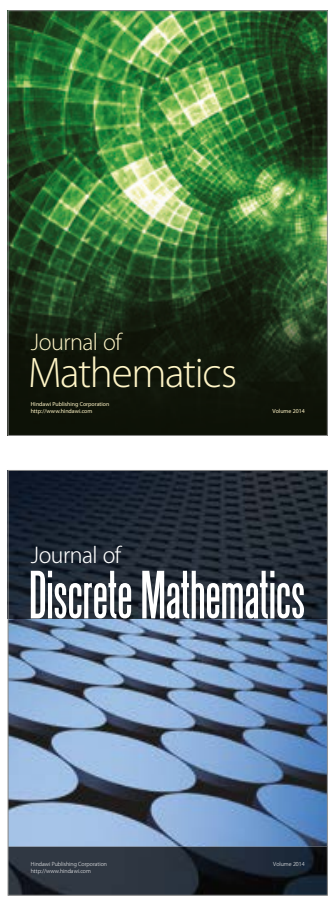

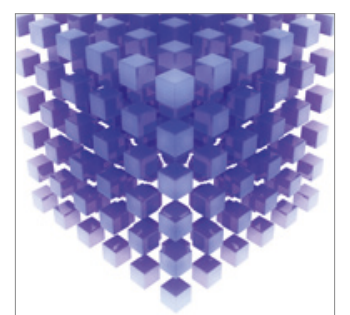

Mathematical Problems in Engineering
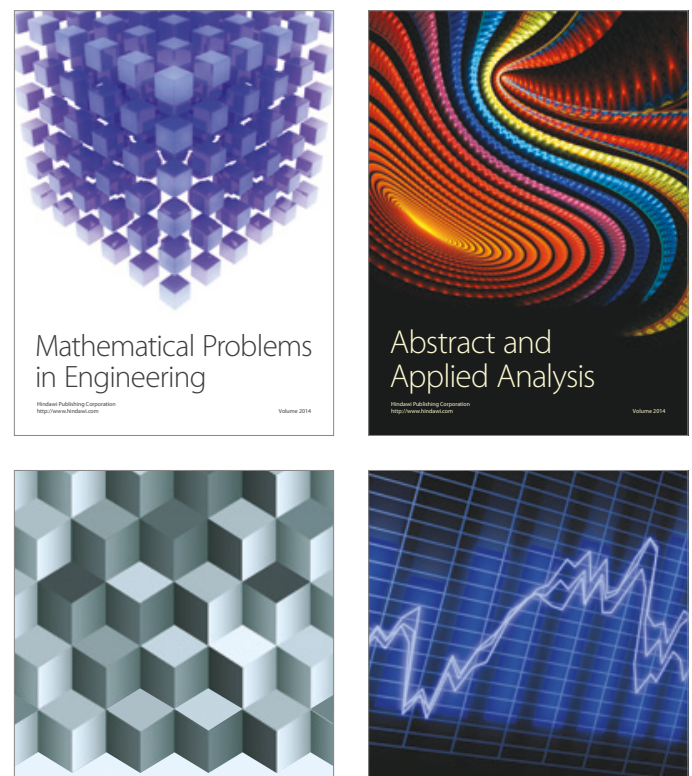

Journal of

Function Spaces

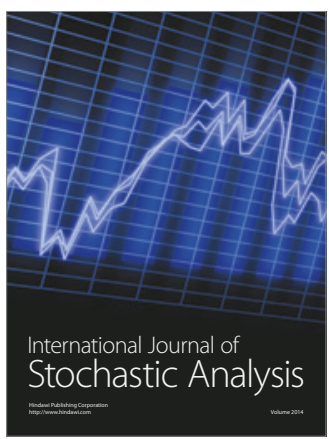

Probability and Statistics
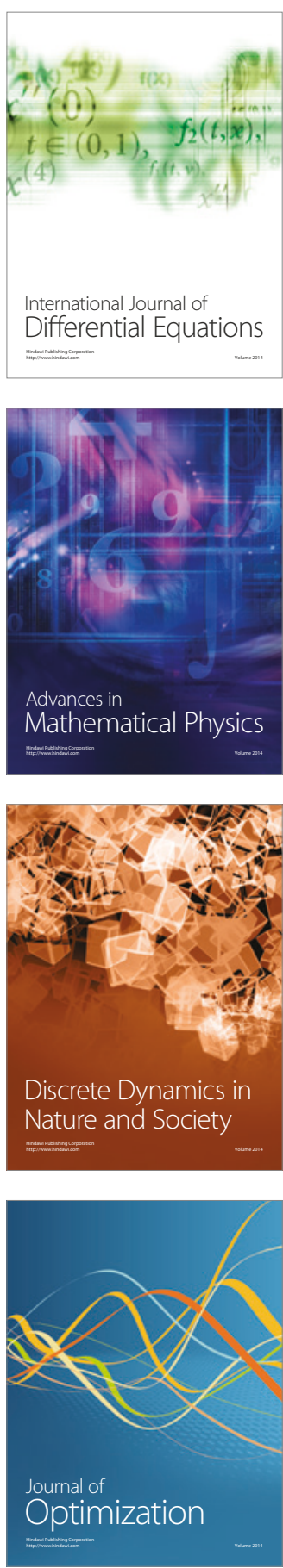